\title{
sciendo
}

RESEARCH PAPERS FACULTY OF MATERIALS

SCIENCE AND TECHNOLOGY IN TRNAVA

SLOVAK UNIVERSITY OF TECHNOLOGY

IN BRATISLAVA

2020, Volume 28, Number 46

DOI 10.2478/rput-2020-0006

\section{MANAGERTYPEN AUS THEORETISCHER SICHT}

\author{
Christine ASCHBACHER ${ }^{1,3}$, Benjamin SCHMACHER $^{2,3}$, Jozef SABLIK ${ }^{3}$ \\ ${ }^{1}$ BundeSMinisterium FÜR ARBEIT, FAMILIE UND JUGEND, \\ UNTERE DONAUSTRAßE 13-15, 1020 WIEN, AUSTRIA \\ ${ }^{2}$ SMS AUSTRIA GMBH, SMS-STRAßE 1, 9065 EBENTHAL IN KÄRNTEN, AUSTRIA \\ ${ }^{3}$ SLOVAK UNIVERSITY OF TECHNOLOGY IN BRATISLAVA \\ FACULTY OF MATERIALS SCIENCE AND TECHNOLOGY IN TRNAVA \\ INSTITUTE OF INDUSTRIAL ENGINEERING AND MANAGEMENT \\ Ulica JÁNA BOTTU 2781/25, 91724 TRNAVA, SLOVAK REPUBLIC \\ e-mail: christine.aschbacher@gmx.at, benjamin.schmacher@sms-group.at, \\ jozef.sablik@stuba.sk \\ Received 15 April 2020, accepted 28 April 2020, published 25 June 2020
}

\begin{abstract}
Abstrakt
Die im Rahmen dieser Veröffentlichung durchgeführten Informationsansammlungen konnten die theoretischen Darstellungen hinsichtlich der Managertypen dargestellt werden. Kurzerhand wurde die erforderliche Theorie zum Thema Managertypen und deren unterschiedlichen Arten erläutert. Ebenso wurden teilweise deren Eigenschaften sowie die Befragungen beigefügt. Abschließend ist zu sagen, dass eine gleichmäßige Verteilung der unterschiedlichen Managertypen für ein ausgewogenes und erfolgreiches System in einem Unternehmen erforderlich sind.
\end{abstract}

\section{Schlüsselwörter}

Betriebsmanagement, Managertypen, Managerkompetenzen, Verteilung der Managertypen

\section{KURZFASSUNG}

Unternehmenserfolge können durch unterschiedliche Managertypen erzielt werden. Die Voraussetzung hierzu ist den richtigen Managertypen dementsprechend im Unter- nehmen einzusetzen [1].

Die Zielfestlegung für das gesamte Team ist nur am Anfang jedes Projektes erforder- lich. In weiterer Folge gibt es eine ganze Reihe von Herausforderungen, welcher sich ein Manager zu stellen hat [2].

Aus diesem Grund werden sieben verschiedene Entwicklungsstufen in den unterschiedlichsten Verhaltensweisen von Führungskräften dargestellt. Im Folgenden wer- den die sieben Managertypen und ihre Handlungsweisen aufgezeigt. Laut Untersu- chungen von Bill Torbert werden sie wie folgt aufgestellt [3]: 
Expert/-e/-in (38\%)

Macher/-in (30\%)

\author{
Diplomat-/in (12\%) \\ Individualis/-int (10\%) \\ Opportunist-/in (5\%)
}

Strateeg/-in (4\%)

Alchimist/-in (1\%)

\section{MANAGERTYPEN - ARTEN DER MANAGERTYPEN}

\section{Der Experte / die Expertin}

Die ExpertInnen werden als die größte Gruppe in der Managementebene gesehen und gewertet. In der Studie von Torbert macht diese Gruppe den Maximalwert von 38 Pro- zent aus. Im Gegensatz zu den OpportunistInnen, die vorrangig versuchen, ihre Um- welt zu beherrschen und zu den DiplomatInnen, die sich darauf konzentrieren, das eigene Verhalten zu kontrollieren, versuchen ExpertInnen, die Kontrolle zu erlangen, indem sie ihr Wissen in beruflicher wie auch in privater Hinsicht perfektionieren.

Argumente sind für die Experten das A und O. Im Speziellen sind für die ExpertInnen wie

- WirtschaftsprüferInnen

- MarktforscherInnen

- IT-TechnikerInnen

- SpeziellstInnen im Bereich Wertpapiere

- UnternehmensberaterInnen

- usw.

außerordentlich wichtig und in der Bewertungsskala ganz weit oben $\mathrm{zu}$ finden. Die Fachkenntnis ist zu 100 Prozent gegeben. ExpertInnen präsentieren ihre Fakten und Zahlen hart und jede dazugehörige Aussage wird logisch und nachvollziehbar abge- geben. Dies wird dazu verwendet, um die KollegInnen für ihre Vorschläge zu gewin- nen. Die kontinuierlichen Verbesserungsvorschläge, Perfektion und Effizienz stellen die ExpertInnen als die perfekter KollegInnen dar.

Hinsichtlich der Führungsqualität sind diese Personen auch von einer anderen Seite zu sehen: Sie sind sich immer absolut sicher und möchten immer Recht haben. Wenn das Sprichwort „Friss oder stirb!““ von dem Kollegium in den Mund genommen wird, spricht man höchstwahrscheinlich von einer Person, die die Handlungslogik eines Ex- perten aufweist. Sie sehen oftmals die Zusammenarbeit mit dem Kollegium als Zeitverschwendung an:

„Nicht alle Treffen sind Zeitverschwendung, manche werden abgesagt!“‘.

Sie nehmen die Meinung von weniger qualifizierten Menschen kaum an und schenken diesen Missachtung. Emotionen sind nicht gewünscht und werden so gut wie nicht geschätzt.

\section{Der Macher / die Macherin}

Alle Personen, welche sich eine/-n Vorgesetzte/-n wünschen, welche/-r nicht gegen sie arbeitet, sondern sie fordert und unterstützt sowie für gute Stimmung im Team sorgt, gibt es positive Nachrichten: 30 Prozent der Führungskräfte sind sogenannte MacherInnen. Diese Führungskräfte sorgen für

- ein positives Arbeitsumfeld und

- konzentrieren sich auf die Ergebnisse.

Natürlich gibt es keine Vor- ohne Nachteile: Dieser Managertyp neigt sehr oft über den Tellerrand hinweg zu sehen. Er besitzen ein weitaus komplexeres Verständnis von der Welt, als andere Typen von Führungskräften. 
MacherInnen sind sehr offen für produktives Feedback und erkennen, dass viele der Ungereimtheiten sowie Konflikte des Geschäfts auf Grund unterschiedlicher Interpretationsweisen entstehen. Sie verfügen über ein hohes Maß an zwischenmenschlichem Verständnis, um Konflikte zu lösen oder das Kollegium zu lenken sowie andere Per- sonen bzw. das Team positiv zu beeinflussen. Die MacherInnen leiten ein Team von ein bis drei Jahren erfolgreich und erzielen damit die Umsetzung neuer Strategien. Dadurch erkennen sie kurz- und langfristige Ziele und haben den Drang, diese auch dementsprechend umzusetzen. Die MitarbeiterInnenfluktuation ist sehr gering und Ma- cherInnen neigen oft dazu, die Verantwortung an die Personen im Team abzugeben, um ein gewisses Maß an Eigenverantwortung zu fördern sowie die Motivation im Team zu stärken. Ihre Umsetzung von Gedanken und Vorstellungen ergeben im Jahr oft ei- nen doppelt so hohen Bruttoumsatz, wie der von den anderen, bereits erwähnten Ex- pertInnen. Sie liegen auch oft auf „Kriegsfuß“ mit diesen Führungskräften. Diese haben Probleme mit den MacherInnen, da sie den Erfolg der MacherInnen nicht verstecken können, obgleich sie sich als bessere ManagerInnen sehen.

\section{Der Diplomat / die Diplomatin}

Die DiplomatInnenen unter den ManagerInnen erschließen sich ihre Umwelt, im Vergleich zu den OpportunistInnen meist auf eine verträglichere Weise. Jedoch können durch ihren extremen Handlungslogismus auch negative Auswirkungen auftreten, spe- ziell, wenn es sich um TopmanagerInnen handelt.

Der Diplomat/ die Diplomatin dient dem Team stets loyal und versucht,

- höher gestellten KollegInnen zu gefallen und

- Konflikte zu vermeiden.

Der Drang die Kontrolle über das eigene Verhalten zu gewinnen, ist eine Widerspiegelung der extremen Handlungslogistik. DiplomatInnen streben auf keinen Fall die Kontrolle über diverse Ereignisse oder ihre Mitmenschen an. Laut der Handlungslogik der DiplomatInnen wird ein/eine Vorgesetzte/-r dauerhafter akzeptiert und hat mehr Einfluss, wenn er/sie die Gruppennormen einhält und seine/ihre täglichen Aufgaben zufriedenstellend ausführt.

Viele Vorzüge bietet das unterstützende Verhalten in Form von einem Miteinander innerhalb eines Teams. DiplomatInnen werden als soziales Bindeglied für die KollegIn- nen gesehen und sorgen dafür, dass das Bedürfnis der Aufmerksamkeit unter dem Kollegium gewahrt wird.

Dies ist sicherlich ein Hauptgrund, warum die Diplomanten hauptsächlich in den unte- ren Managementstufen tätig sind.

Beispiele hierfür sind:

- Kundenservice

- Kundenpflege

- qualifiziertes Personal

- usw.

Es wird vermutet, dass ManagerInnen mit effektiveren Handlungslogistiken wie z.B.: die Strategen, welche im Laufe dieser Arbeit erläutert werden, eine viel bessere Chance haben, im oberen Managementbereich tätig zu sein. Im Gegensatz zu den OpportunistInnen, die versuchen, Konflikte zu ignorieren bzw. zu vermeiden, wollen sie diese geklärt haben. Sie sind möglicherweise oft zu höfflich und zu freundlich und sehen sich nicht in der Lage, dem Kollegium kritisches Feedback zu geben. 
Bedrohung sehen die DiplomatInnen in Veränderungen, welche mit Konflikten behaftet sind. Diese Personen meiden solche Themen zur Gänze, auch wenn sie sich dabei selbst schaden würden.

\section{Der Individualist / die Individualistin}

Der Individualist / die Individualistin kennt sich und weiß genau, dass seine/ihre Vorgangsweisen sowie auch die der anderen HandlungslogikerInnen natürlich und diverse Konstruktionen aus der Welt und anderer Personen sind. Ein geringer Teil der individualistischen ManagerInnen, bezeichnet diese jedoch als abstrakte Ideen und nicht als natürliche Handlungen. KollegInnen, die andere, unterschiedliche Persönlichkeits- strukturen aufweisen, haben und anderen Handlungslogiken folgen, kommen gut mit IndividualistInnen aus.

IndividualistInnen unterscheiden sich von MacherInnn durch das Bewusstsein, dass es einen Konflikt zwischen ihren Prinzipien und ihren Taten geben kann oder zwischen Unternehmenswerten und der Umsetzung dieser Werte.

Der bereits erwähnte Konflikt ruft die Quellen

- Spannung und

- Kreativität hervor.

Daraus ergibt sich der Wunsch, sich ständig weiterzuentwickeln. Sie neigen auch dazu, die vorgegeben Regeln „links“ liegen zu lassen und ihnen keine Beachtung zu schenken. Es handelt sich dabei meist um Regeln, welche sie als irrelevant ansehen. Das Ignorieren dieser Regeln kann jedoch auch zu Konflikten mit dem Kollegium sowie den Vorgesetzten führen.

\section{Der Opportunist / die Opportunistin}

Laut diversen Studien weisen nur 5 Prozenten der befragten ManagerInnen Eigenschaften wie

- Misstrauen,

- Egozentrik oder

- Bestechlichkeit auf.

Diese Managertypen werden als sogenannte OpportunistInnen bezeichnet.

Diese Bezeichnung spiegelt die Tendenz zum eigenen Vorteil wieder. Das bedeutet, dass diese Personen nach Gelegenheiten suchen, um ihre Mitmenschen und die Welt auszunutzen. Die Umwelt wird unter dem Aspekt der Eigenkontrolle betrachtet - In- wiefern habe ich die Kontrolle?

Grundsätzlich ist damit gemeint, dass ihre Entscheidungen davon abhängen, ob sie Einfluss auf das Ergebnis haben oder nicht. Die Mitmenschen werden mehr als diverse Objekte oder KonkurrentInnen gesehen. OpportunistInnen leben nach dem Motto „Auge um Auge, Zahn um Zahn“" und sehen dies als legitim an.

Feedback wird grundsätzlich abgelehnt und die Schuld wird immer bei den Anderen gesucht. Darüber hinaus, ist der Drang zum Ausüben grausamer Rache, sehr ausge- prägt.

Befragte ManagerInnen bezeichnen ihren Managementstil zu Beginn der Karriere als „Management by Verhöhnung“... Nur wenige OpportunistInnen halten sich lange als ManagerInnen, es sei denn, sie gehen zu effektiveren Handlungslogiken über.

Viele Personen wollen auf Grund der ständigen Konfrontationen, der Selbstverherrli- 
chung und der häufigen Regelverstöße der OpportunistInnen mit solchen Führungs- kräften nicht lange zusammenarbeiten. Sofern man für OpportunistInnen gearbeitet hat, wird dies eine Zeit gewesen sein, welche durch viele Differenzen und Schwierig- keiten in Erinnerung bleiben wird.

Der Opportunismus findet meist in Unternehmen Platz, welche keine lange Lebens- zeit aufweisen. Immerhin bieten OpportunistInnen ein spannendes Umfeld, vor allem für jüngere, risikobereite Führungskräfte.

\section{Der Stratege / die Strategin}

Führungskräfte mit den Eigenschaften, organisatorische Zwänge und Wahrnehmun- gen als diskussionswürdig und veränderbar anzusehen, bezeichnet man als StrategIn- nen. Im Gegensatz zu IndividualistInnen können die StrategInnen auf einer überge- ordneten Ebene die Auswirkungen von Maßnahmen und Vereinbarungen auf das Un- ternehmen einschätzen, wohingegen die IndividualistInnen die Kommunikation mit den unterschiedlichsten Handlungslogiken bevorzugen und beherrschen.

Die Entwicklung von gemeinsamen Visionen für ManagerInnen mit den unterschiedlichsten Handlungslogiken, ist das Steckenpferd von den StrategInnen. Eine soziale und organisatorische Veränderung brauchen einen längeren Entwicklungsprozess, was nicht jeder Führungskraft so im Bewusstsein ist, jedoch eingeräumt werden muss. StrategInnen fühlen sich wohl dabei, etwas Gutes zu tun und zur Lösung von Konflik- ten beizutragen. Ebenfalls können sie sehr gut mit Veränderungen der Menschen um- gehen. Das sieht ein Kollegium mit anderen Vorlieben ganz anders. Dies ist der Grund, warum StrategInnen hocheffiziente ErneuerInnen sind.

\section{Der Alchimist / die Alchimistin}

Der letzte Managertyp im Bunde ist der Alchimist/ die Alchimistin. Nachforschungen über die Manager, die als AlchimistInnen identifiziert wurden, gehen davon aus, dass der Unterschied zu den StrategInnen ihre Fähigkeit ist, sich selbst oder das betref- fende Unternehmen auf historisch bedeutsame Weise zu erneuern oder, ihnen sogar eine völlig neue Ausrichtung zu geben. Der Alchimist/die Alchimistin besitzt die Fähig- keit, auf den unterschiedlichsten Ebenen erfolgreich zu sein. Er/sie ist als Führungs- kraft genauso gut und sensibel wie ein Kollegium aus dem Team. Kurzfristige Ziele können rasch und sorgsam erledigt werden, wobei das langfristige Ziel aus in keiner Weise aus den Augen verloren wird. Die geringe Prozentzahl von eins deutet darauf hin, dass diese Managertypen sehr selten anzutreffen sind.

Ebenfalls kümmern sich diese Personen in Unternehmen um mehrere Aufgaben und finden dennoch die Zeit, kurzfristige Aufgaben, welche zügig erledigt werden müssen, zum Abschluss zu bringen. Der Stresspegel dieser Personen war gar nicht wahrge- nommen.

AlchimistInnen sind in der Regel

- charismatische und

- extrem aufmerksame Persönlichkeiten.

Sie leben ein Leben mit hohen moralischen Maßstäben und legen sehr viel Wert auf die Wahr- und Ehrlichkeit. Am wichtigsten ist, dass diese Personen in der Lage sind, die Einzigartigkeit der Unternehmensgeschichte zu nutzen und Symbole und Meta- phern zu schaffen, die die Herzen und den Geist der Menschen ansprechen. 


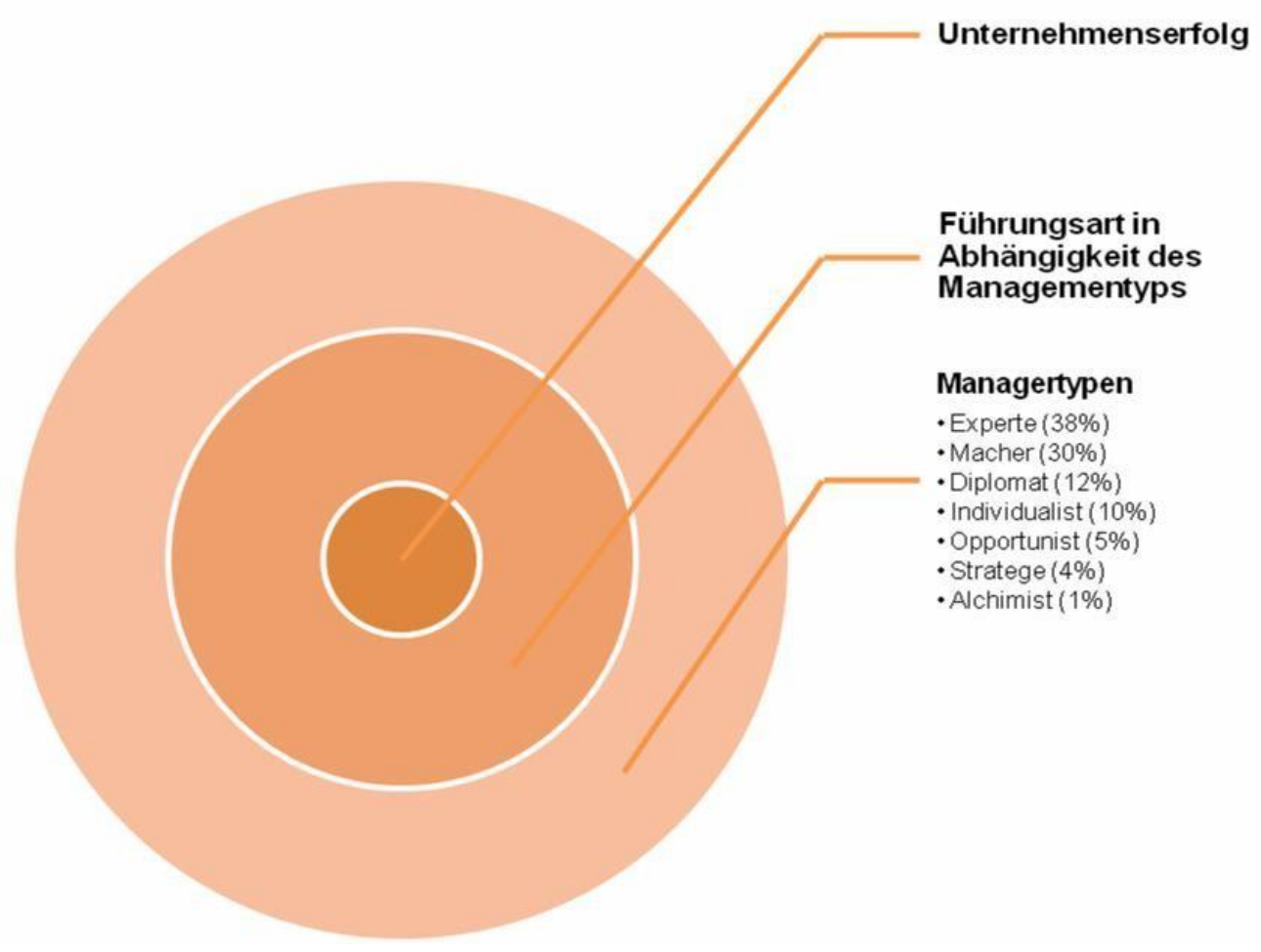

Abbildung 1. Eigene Darstellung - Einfluss des Unternehmenserfolges durch Managertypen [4]

\section{SCHLUSSWORT}

Aus dieser Veröffentlich wird der Entschluss gezogen, dass es erforderlich ist, zuerst Begriffe zu erläutern und deutlich darzustellen, bevor weitere Schritte vorgenommen werden. Dies dient zum leichteren Verständnis, dass für ein gesundes Unternehmen diverse und vorallem mehrere Managertypen vorhanden sein sollten.

\section{Literaturverzeichnis}

Monographien und sonstige selbständige Veröffentlichungen

[1] BENDER Susanne. 2015. Teamentwicklung: Der effektive Weg zum „Wir“. dtv Verlagsgesellschaft. Letzte Auflage. ISBN: 978-3-423-50945-9

[2] NIERMEYER Rainer. 2016. Teams führen. Haufe-Lexware GmbH \& Co. KG, Letzte Auflage. ISBN: 978-3- 648-08447-2

[3] BENJAMIN, A., SCHMACHER, K. 2016. Mitarbeitermotivation anhand der Winterbereifung. Saarbrücken: AV Akademikerverlag, ISBN 978-3-639-87879-0

[4] ÖTTL Christine, HÄRTER Gitte 2015. Vorstellungsgespräche. München: GU Verlag, ISBN 3-7742-6385-x

[5] DEIBL Maria. 2015. Sind Sie ein idealer Chef. Wien: P. Linde-Verlag, 1990. ISBN 385122-279-2

Internetquellen und übriges Informationsmaterial

[6] DI. Dr. Michael Paula Unternehmensberatung (2017). Transformative Führung - die sieben Managertypen (2/2) von Michael Paula, online im Internet: http://www.walkyourtalk.at/transformative-fuehrung-die-sieben- managertypen-22/leadership/, 14. Dezember 2017. 